\title{
Frequency of Hydrocephalus in Patients with Chronic Meningitis due to Mycobacterium Tuberculosis
}

\author{
Mumtaz Ali ${ }^{1}$, Adnan $\mathrm{Khaliq}^{2}, \mathrm{Farooq}_{\mathrm{Azam}}{ }^{3}, \mathrm{Amin}$ ul Haq ${ }^{4}, \mathrm{Nayab}^{\mathrm{Gu}}{ }^{5}$
}

\section{ABSTRACT}

Background: Tuberculosis is one of the causes of chronic meningitis which may lead to hydrocephalus. Uncertainty and doubt dominate all aspects of tuberculous meningitis.

Objectives: To determine the frequency of hydrocephalus in tuberculous meningitis patients.

Material \& Methods: This was observational descriptive study conducted at department of neurosurgery and neurology lady reading hospital Peshawar from Jan,2016 to Dec,2018. In this study there were total 93 cases of TBM with $95 \%$ confidence level,2.2\% margin of error according to WHO calculator. Diagnosis was based on clinical features, CSF analysis and CT/MRI brain. Tuberculous meningitis patients of age range 15 to 50 years of either gender were included in this study. All those patients having SOL in brain and other causes of meningitis were excluded from this study. The symptoms were fever, headache, and weight loss and neck rigidity with or without fits. These cases then underwent CSF analysis under aseptic measures. The findings to label TBM on CSF were low glucose $(<40 \mathrm{mg} / \mathrm{dl})$, increased protein content $(>100 \mathrm{mg} / \mathrm{dl})$ and high WBC count with predominance of lymphocytes. The data was analyzed with the help of SPSS version 23 .Effect modifiers were stratified and and post stratification Chi-Square test was applied with P-value $<0.05$ as significant. The cases of TBM were divided according to standard BMRC scale into three stages.

Results: In this study there were total 93 cases with mean age of $37.11 \pm 08.67$ years. There were $54(58.06 \%)$ males and 39 $(41.94 \%)$ females. Maximum cases were seen in stage II of TBM which affected $65(69.89 \%)$ cases. Hydrocephalus was seen in 61 $(65.59 \%)$ of the cases. Hydrocephalus was significantly high in male gender as compared to females where it affected $39(72.22 \%)$ of cases. It was also more common in cases that had age group 15-29 years affecting $40(67.79 \%)$ of cases.

Conclusion: Hydrocephalus is a common complication of tuberculous meningitis and it is predominantly seen in in male patients, stage II and III of TBM.

Keywords: TBM, Hydrocephalus, Meningitis.

This article may be cited as: Ali M, Khaliq A, Azam F, Haq AU, Gul N.

Frequency of Hydrocephalus in Patients with Chronic Meningitis due to Mycobacterium Tuberculosis. J Saidu Med Coll Swat 2021;11(1):03-08

\section{INTRUDUCTION}

Tuberculosis (TB) is a disease of the ancient times and still it exists, about 2000 million people in the world today are infected with tuberculosis ${ }^{1}$. The number are highest in the developing countries and its incidence rate is 275 per 100,000 population in Pakistan according to World Health Organization ${ }^{1}$. Tuberculosis usually involves lungs but it can involve any part of the body and is a great mimicker of wide range of disease, hence leading to delay in diagnosis and worst outcomes. Most of the patients infected with mycobacterium tuberculosis are clinically asymptomatic and few presents with symptoms. Why some people develop clinical disease remains unclear. The reasons are likely multifactorial including genetic predisposition, comorbid conditions and environmental factors. Risk factors identified in adults are alcoholism, diabetes mellitus,

1. Department of Neurosurgery, Prime Teaching Hospital/Peshawar Medical College.

2. Department of Neurosurgery, Saidu Medical College/Saidu Teaching Hospital, Swat.

3. Department of Neurosurgery Lady Reading Hospital Peshawar.

4. Department of Medicine, Saidu Teaching Hospital Swat.

5. Rehman Medical Institute/Rehman Medical College, Hayatabad Peshawar.

Correspondence: Dr Adnan Khaliq

Department Of Neurosurgery

Saidu Medical College/Saidu Teaching Hospital,Swat.

Email: dradnankhaliq@gmail.com malignancy, HIV infection and corticosteroid use $^{3}$.In children risk factors identified are poverty, and malnutrition ${ }^{4}$.The extent to which a person genetic constitution affects resistance or susceptibility to infection in under debate. Certain ethnic groups like black skinned people are more susceptible to infection than white people $e^{4,5}$. The extent to which BCG vaccination provides protection against TBM is still debated. A meta analysis of published trials showed BCG vaccination protective effect of $64 \%$ against $\mathrm{TBM}^{6}$.Keeping in view this and other published articles support that $B C G$ vaccination is protective against $\mathrm{TBM}^{6}$.It can involve brain in the form of meningitis, encephalitis and tuberculoma ${ }^{2}$.Tuberculous meningitis was first described as distinct pathological entity in $1936^{2}$ caused by mycobacterium tuberculosis an aerobic gram positive rods that stains poorly due to its thick cell wall containing lipids, peptideglycans.

Uncertainty dominate all aspects of tuberculous meningitis (TBM).Variable natural history and accompanying clinical features hinders diagnosis. Delayed diagnosis and missed treatment can result in fatal outcomes ${ }^{3}$. According to studies ${ }^{7,8}$ the development of TBM is 
considered two step process. Initially tuberculous bacilli enter the host by droplet inhalation, the initial point of infection being the alveolar macrophages. Escalating localized infection within the lung with dissemination to the regional lymph nodes produces primary complex. During this stage there is short but significant bacteremia that can seed tubercle bacilli to other organs in the body. In those who develop TBM, bacilli seed to the meninges or brain parenchyma ${ }^{8,9}$. Dissemination to the CNS is more likely if military TB develops. The second stage of TBM is rupture of CNS foci into subarachnoid space. This heralds the onset ofmeningitis ${ }^{9,10}$.

Three general processes produce the subsequent neurological pathology. Adhesion formation, obliterative vasculitis and encephalitis ${ }^{11}$. Adhesions result from a dense meningeal exudate that develops after inoculation of bacilli into subarachnoid space. The exudate contains lymphocytes, plasma cells, macrophages with increasing quantities of fibrin. Blockage through adhesion of the basal subarachnoid cisterns and aqueduct of sylvius can result in obstruction of the CSF and hydrocephalus. An obliterative vasculitis of both large and small vessels develop that can result in ischemic stroke and irreversible neurological sequelae. Basal inflammatory process extends into the parenchyma resulting in encephalitis ${ }^{12,13}$.

The clinical presentation of TBM is variable. Diagnosis on clinical grounds is not possible. Recent contact with a tuberculous patient is seen in $70 \%$ to $90 \%$ patients. The prodrome is usually non specific, $28 \%$ report headache, $25 \%$ vomiting, $13 \%$ had fever. Only $2 \%$ reported meningitis symptoms ${ }^{14,15}$. The neurological complications that can occur are cranial nerves palsies $\left(3^{\text {rd }}, 4^{\text {th }}, 6^{\text {th }}, 7^{\text {th }}, 8^{\text {th }}\right)$ and hydrocephalus. Hydrocephalus presents with signs and symptoms of aised ICP like Headache, Nausea/Vomiting, Decreased conscious level and sometimes seizures ${ }^{16}$. Infarcts occur in about $30 \%$ of cases commonly in the internal capsule and basal ganglia causing a range of disorders from hemiparesis to movements disorders ${ }^{17}$.

Diagnosis of TBM is dependent on lumbar puncture and CSF examination. Ideally a large volume $(10 \mathrm{ml}$ is recommended) of CSF is collected. Acid fast bacilli are seen in CSF smears in about $10 \%$ to $20 \%$ of those with TBM. Lumbar functure can be repeated in doubtful cases. The success of test depends on the quality and volume of sample sent, the skill of the technician and their persistence in examining the sample. Lymphocytosis of between 100 and 1000 cells $/ \mathrm{mm}^{3}$ is more usual, although in the first 10 days polymorphonuclear leucocytes may predominate. Araised CSF protein occurs in most and CS glucose will be reduced in $70 \%^{18}$. The culture of mycobacterium tuberculosis from the CSF is the GOLD STANDARD for the diagnosis but insensitive and slow. Culture in solid medium such as Lowenstein-Jensen may take upto 8 weeks to culture $M$ tuberculosis. The advent of CT and MRI brain has provided insight into disease progression and gives prognostic and diagnostic information. Both CT and MRI brain with contrast will disclose hydrocephalus, basilar meningeal thickening, infarct, edema and tuberculomas ${ }^{19}$. Hydrocephalus was reported in $87 \%$ of children and $12 \%$ of adults. The incidence is greater in young patients and longer the duration of illness ${ }^{19}$.Tuberculomas appear as ring enhancing lesion with surrounding edema, may be single or multiple. Basal meningeal enhancement is more pronounced in MRI brain. The major role of neuroradiology has been in management and in particular in the diagnosis and follow up of those complications requiring neurosurgery consultation ${ }^{20}$.

Treatment of uncomplicated TBM is chemotherapy with ATT. The current United kingdom guidelines ${ }^{21}$ suggest treatment for the first two months with rifampicin $(20 \mathrm{mg} / \mathrm{kg})$, Isoniazid $(20 \mathrm{mg} / \mathrm{kg})$, Ethambutol $(20 \mathrm{mg} / \mathrm{kg})$, Pyrazinamide $(40 \mathrm{mg} / \mathrm{kg})$ for 12 months. The initial phase of treatment for 2 months including all the four drugs and later 10 months include 2 drugs(INH and RIF). Steroids in

Table 1 British Medical research council(BMRC) staging of tuberculous meningitis ${ }^{15}$

\begin{tabular}{|c|c|}
\hline Stage & Status of patient \\
\hline $\mathbf{1}$ & Fully conscious,No paresis \\
\hline $\mathbf{2}$ & Decreasing conscious level,Localising pain \\
\hline $\mathbf{3}$ & Deeply comatosed/Gross paresis \\
\hline
\end{tabular}


the form of Prednisolone at a dose of $4 \mathrm{mg} / \mathrm{kg}$ is also used which has beneficial effects of reducing edema, Infiltration of antibiotics to CNS and reducing the harmfull effects of cytokine release to CSF after killing of bacteria ${ }^{22}$.

The role of neurosurgeon starts with neurological deterioration in a patient under treatment for TBM. There are various causes of this and require radiological assessment. There may be hydrocephalus or tuberculoma with surrounding edema on CT/MRI brain. Hydrocephalus is a common complication that may lead to permanent neurological damage or death if left untreated ${ }^{23}$. The prevalence of hydrocephalus ranges from 20$65 \%{ }^{3-4}$. The data from Pakistan has shown its range from $58 \%$ in a study from Rawalpindi to $72.3 \%$ in Karachi.$^{5-6}$ However one study from India on 45 cases revealed this hydrocephalus in 33.3 $\%$ of cases only ${ }^{7}$. Prompt assessment by CT brain is mandatory both for early diagnosis and management. Studies suggest that prompt ventriculoperitoneal shunting improve outcome ${ }^{24}$. Tuberculoma is treated conservatively with ATT along with steroids and most of these show good resolution on follow up images ${ }^{22}$.

\section{MATERIALS AND METHODS}

This was an observational descriptive study with non probability consecutive sampling technique. This study was conducted at department of Neurosurgery And Neurology Lady Reading Hospital Peshawar from Jan, 2016 to Dec,2018.In this study there were total 93 cases of TBM with $95 \%$ confidence level,2.2\% margin of error according to WHO calculator. The cases of tuberculous meningitis of age range of (15 to 50) years of either gender were included in this study. The cases with bacterial meningitis, having any SOL in brain and those with any previous history of neurosurgery were excluded from this study. The diagnosis of TBM and hydrocephalus was made on the basis of combination of clinical features, laboratory investigations and CT/MRI brain. The Clinical features were Headache, fever, nausea/vomiting, neck rigidity with or without fits. In cases of hydrocephalus papilledema was found. These cases then underwent lumbar puncture under aseptic measures for CSF analysis. The findings to label TBM on CSF were low glucose $(<40 \mathrm{mg} / \mathrm{dl}$ ), increased protein content $(>40 \mathrm{mg} / \mathrm{dl})$ and high WBC count with predominance of lymphocytes. Patient with clinical suspicion of hydrocephalus were screened through CT brain and then MRI brain for more detailed description. Radiological features of hydrocephalus according to Evan's ratio were dilatation of lateral ventricles, ballooning of third ventricle(More than $5 \mathrm{~mm}$ ), Enlargement of temporal horns(More than 2mm), Periventricular lucency, Obliteration of sulci and sylvian fissures, Effacement of basal cisterns, Most of the cases showed triventricular hydrocephalus(Dilataion of lateral and 3rd ventricles) but some patients showed tetraventricular hydrocephalus. All the patients diagnosed as hydrocephalus were treated by Ventriculoperitoneal shunt and post operartive ATT for one year. Follow up neuroimaging was done after 6 months. The cases of TBM were divided according to standard BMRC scale into three stages. The data was analyzed with the help of SPSS version 23. Effect modifiers were stratified and and post stratification Chi-Square test was applied with $P$-value $<0.05$ as significant.

\section{RESULTS}

In this study there were total (93) patients of TBM with mean age of $(37.11 \pm 08.67)$ years. There were $54(58.06 \%)$ males and 39 (41.94\%) females. Maximum cases were seen in stage II of TBM which affected $65(69.89 \%)$ cases. Hydrocephalus was seen in $61(65.59 \%)$ of the cases as shown in figure 01 . Hydrocephalus was significantly high in male gender $39(72.22 \%)$ as compared to females $22(56.41 \%)$ with $p$ value of 0.03 . It was also more common in cases that had age group 15-29 years affecting $40(67.79 \%)$ of cases with $p=0.86$.Hydrocephalus was also significantly high in cases with stage II and III of TB affecting $66.15 \%$ and $77.27 \%$ of cases respectively with $p=0.01$. We found tetraventricular hydrocephalus in 39 cases $(63.9 \%)$ and triventricular hydrocephalus in 21 cases $(34.4 \%)$.

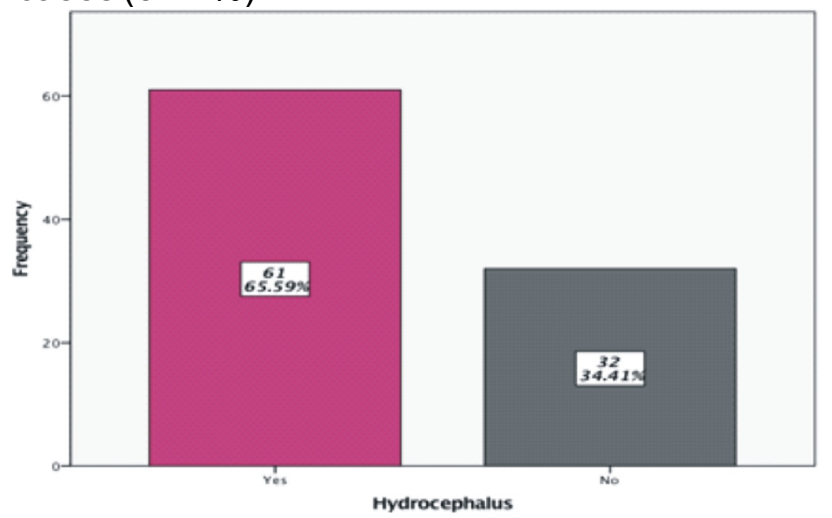

Figure I Hydrocephalus in TBM 
Table 2. Clinical presentation of TBM and hydrocephalus

\begin{tabular}{|c|c|c|}
\hline Symptoms and signs & Number of patients & Percentage \\
\hline Fever & 32 & $34.4 \%$ \\
\hline Headache,Nausea,Vomiting & 30 & $32.2 \%$ \\
\hline Signs of meningeal irritation & 30 & $32.2 \%$ \\
\hline Altered sensorium & 17 & $18.2 \%$ \\
\hline Cranial nerve palsy & 29 & $31.1 \%$ \\
\hline Papilledema & 61 & $65.5 \%$ \\
\hline Neurodeficits & 32 & $34.4 \%$ \\
\hline Fits & 23 & $24.7 \%$ \\
\hline Anemia & 19 & $20.4 \%$ \\
\hline
\end{tabular}

Table 3. Hydrocepahalus and Stages of TBM

\begin{tabular}{|c|c|c|c|}
\hline \multirow{2}{*}{ Stage of TBM } & \multicolumn{2}{|c|}{ Hydrocephalus } & \multirow{2}{*}{ Total } \\
\cline { 2 - 4 } & Yes & No & $06(100 \%)$ \\
I & $01(16.67 \%)$ & $05(83.33 \%)$ & $65(100 \%)$ \\
II & $43(66.15 \%)$ & $22(33.85 \%)$ & $22(100 \%)$ \\
III & $17(77.27 \%)$ & $05(22.73 \%)$ & $\mathbf{9 3 ( 1 0 0 \% )}$ \\
\hline
\end{tabular}

\section{DISCUSSION}

Tuberculosis continues to be a a major health problem for developing and poor countries. One third of world population are infected with mycobacterium tuberculosis ${ }^{4}$. The most common form of TB is pulmonary and the most dangerous form is that of CNS accounting for $5.2 \%$ of clinical TB and almost $50 \%$ morbidity $^{6}$. Hydrocephalus is the most common complication of TBM .It is almost always present in patients who have had the disease for four to six weeks. shoeman at al found the hydrocephalus was $65 \%$ in his study ${ }^{24}$. In our study we found $61 \%$ patients that are diagnosed with TBM developed hydrocephalus. It is more frequent and severe in children and early adulthood ${ }^{21}$. This has been seen in our study too, where the majority of patients in early adulthood (15-29) years showed post TBM hydrocephalus. Hydrocephalus was significantly high in male gender as compared to females where it affected $39(72.22 \%)$ of cases with $p$ value of 0.03 . Male gender was also proved as a risk factor for the development of hydrocephalus in cased of TBM by previous studies. Kumar and Christensen AS et al found that males were seen in more than $2 / 3^{\text {rd }}$ cases of TBM; though this difference was not statistically significant with $p$ values of 0.54 and 0.34 resepctively ${ }^{12-13}$. Hydrocephalus was also significantly high in cases with stage II and III of TB affecting $66.15 \%$ and $77.27 \%$ of cases respectively with $p=0.01$. The studies have shown that there is liner association of the severity of the disease and the development of the hydroecephalus. ${ }^{14-15}$ Chan et al in their study found $89 \%$ of the cases to develop hydrocephalus in cases of TBM. ${ }^{11}$ The reason can be explained by the fact that the severe the disease and higher is the turbidity of the CSF and led to difficult drainage and ultimately led to hydrocephalus.

Hydrocephalus in patients could be either of communicating type or obstructive type ${ }^{25}$,the former being more common. This has been demonstrated in our study too showing (63.9\%) patients communicating type hydrocephalus. In both instances the main cause is the inflammatory exudate occupying the subarachnoid spaces or the ventricular pathways. In the earlier stages of disease, the thick gelatinous exudates block the subarachnoid spaces in the base of the brain(Interpedicular and ambient cisterns) leading to communicating type hydrocephalus ${ }^{26}$. The exudates lead to a dense scarring of the subarchnoid spaces in the later stages of the disease again leading to communicating type of hydrocephalus. A communicating hydrocephalus can also result from the exudates blocking the arachnoid granulation which prevent the absorption of cerebrospinal fluid(CSF). The obstructive type of hydrocephalus develops when there is obstruction of the aqueduct or fourth ventricular 
outlets by exudates ${ }^{27}$. The clinical features that suggest the presence of hydrocephalus are nonspecific. In any patient with TBM with altered sensorium, hydrocephalus should be suspected irrespective of the presence or absence of papilledema. Hydrocephalus is also likely to be present in patients who are alert and who complain of increasing headache with or without vomiting and blurring of vision. Although a widely used grading system exists for patients with TBM, namely MRC grading system, a specific grading system did not exist for patients with TBM and hydrocephalus. Another proposed grading system for TBM and hydrocephalus is (Vellore grading system) based on the presence or absence of neurological deficits and level of sensorium $^{15}$.

A contrast enhanced CT scan brain is the imaging of choice in sick patients as it can performed without anesthesia or sedation and can be done in a short time compared to MRI brain which might require a patient to still lie for longer periods. Adequate information regarding ventricular size, subependymal seepage, presence of infarcts, tuberculomas and presence of basal exudates can be obtained from a contrast enhanced CT scan. ACT scan brain may indicate whether the hydrocephalus is obstructive or communicating in nature.

For physicians, when they encounter a patient with post TBM hydrocephalus, they should start with medical management as first line of theraphy. This has been mentioned in literature ${ }^{10}$.Medical management comprises of ATT, steroids and dehydrating agents such as mannitol, furosemide or acetazolamide. Tapering dose of dexamethasone(12 to $16 \mathrm{mg} /$ day) can be given for four to six weeks. Mannitol can be used for acute decompensation and is not recommended for more than 72 hours due to rebound intracranial hypertension. Acetazolamide $(100 \mathrm{mg} / \mathrm{kg})$ and furosemide $(1 \mathrm{mg} / \mathrm{kg})$ can be given for longer periods upto a month. Medical management is generally successful in several patients with TBM who exhibit features of raised intracranial pressure. Along with agents mentioned above, Antituberculous theraphy(ATT) also probably helps in reducing the inflammatory response leading to opening of the CSF pathways. The addition of acetazolamide and furosemide was significantly effective in achieving normal Intracranial pressure(ICP) than ATT alone ${ }^{22}$.In a study from south Africa by Lamprect et al $^{10} 217$ children with MRC stage 2/3 TBM and hydrocephalus were initially managed medically if they had communicating hydrocephalus. This strategy allowed the authors to avoid shunt surgery in over $70 \%$ of patients. In other $30 \%$ patients, who underwent surgery had obstructive hydrocephalus. So it appears prudent to suggest medical management for patients with better sensorium and communicating hydrocephalus. However medical management implies continuous monitoring of patients. Patients with obstructive hydrocephalus and acute deterioration need urgent shunt surgery as delayed treatment lead to poor outcome.

Ventriculo-atrial(VA) shunts were the initial choice for shunt surgery. The fear of disseminating the tuberculous disease through such shunt system was dispelled by reports ${ }^{22-24,26,28}$,who described the successful use of VAshunts in patients with TBM. However some reported the occasional dissemination of the disease following VA shunts in patients with $\mathrm{TBM}^{27}$.So there was a shift to ventriculoperitoneal shunt in the early 1980s and since then VP shunt has remained the procedure of choice for patients with TBM and hydrocephalus. Although there is a theoretical risk of developing tuberculous peritonitis following VP shunt, literature review has not showed such an occurrence. The technique for shunt surgery does not differ from that used for hydrocephalus from any other cause. Outcome of surgery depends on clinical factors like age, duration of illness, sensorium of patients. Biochemical factors predicting outcome are CSF protiens count, CSF cell count, Basal ganglia, internal capsule infarcts are also poor predictors of outcome ${ }^{21}$.

\section{CONCLUSION}

Hydrocephalus is a common complication of TBM and it is frequently seen in males, $2^{\text {nd }}$ and $3^{\text {rd }}$ decades of life and stage $2 /$ stage 3 of TBM. Timely management leads to a better outcome.

\section{REFERENCES}

1. DeLance AR, Safaee M, Oh MC, ClarkAJ, Kaur G, Sun $\mathrm{MZ}$, Bollen AW. Tuberculoma of the central nervous system: J Clinic Neurosci 2016,20(10):1333-1341.

2. Garg RK, Malhotra HS, Kumar N. Paradoxical reaction in HIV negative tuberculous meningitis: J Neuro Sci 2018,340(1-2):26-36.

3. Afghani B, Lieberman JM. Paradoxical enlargement or development of intracranial tuberculomas during therapy: J Clin Infect Dis 2017,19(6):1092-1099.

4. Nicolls DJ, King M, Holland D, Bala J, del Rio C. Intracranial tuberculomas developing while on therapy for pulmonary tuberculosis. Lancet Infect Dis 2015,5(12):795-801. 
5. Cheng VC, Ho PL, Lee RA, Chan KS, Chan KK, Woo PC, Lau SK. Clinical spectrum of paradoxical deterioration during antituberculosis therapy in nonHIV-infected patients:J Eue Clin Microbiol Infect Dis 2018, 21(11):803-809.

6. Ranjan P, Kalita J, Misra. Serial study of clinical and CT changes in tuberculous meningitis: $J$ of Neuroradio 2018,45(5):277-282.

7. Anuradha HK, Garg RK, Sinha MK, Agarwal A, Verma $\mathrm{R}$, Singh MK. Intracranial tuberculomas in patients with tuberculous meningitis: predictors and prognostic significance: Int J Tuberc Lung Dis 2019,15(2):234239.

8. Singh AK, Malhotra HS, Garg RK, Jain A, Kumar N, Kohli N, Verma. Paradoxical reaction in tuberculous meningitis: presentation, predictors and impact on prognosis: J BMC Infect Dis 2016, 16:306-12.

9. Kalita J, Prasad S, Misra UK. Predictors of paradoxical tuberculoma in tuberculous meningitis: Int $\mathrm{J}$ Tuberc Lung Dis 2018, 18(4):486-491.

10. Sutlas PN, Unal A, Forta $H$, Senol S, Kirbas D. Tuberculous meningitis in adults: review of 61 cases: J of CNS Infection 2018,31(6):387-391.

11. Tai ML, Nor HM, Kadir KA, Viswanathan S, Rahmat K, Zain NR, Ong KG Paradoxical Manifestation is Common in HIV-negative Tuberculous Meningitis: JNS 2016,95(1):80-86.

12. Thwaites GE, Macmullen-Price J, Tran TH, Pham PM, Nguyen TD, Simmons CP. Serial MRI to determine the effect of dexamethasone on the cerebral pathology of tuberculous meningitis: an observational study:J Lancet Neurol 2017, 6(3):230-236.

13. Thuong NTT, Thwait. Treatment-As so ciated Inflammatory Deterioration in Tuberculous Meningitis, Unpicking the Paradox:J Infect Dis 2017,215(5):665667.

14. Chen F, Chen L, Cao Y, Yi Y, Zhuang J, Le W, Xie W, Tu L, Li P. Intracisternal tuberculoma:a refractory type of tuberculoma indicating surgical intervention: BMC Neurol 2018, 18(1):10-13

15. Cheng VC, Yam WC, Woo PC, Lau SK, Hung IF, Wong SP, Cheung WC. Risk factors for development of paradoxical response during antituberculosis therapy in HIV-negative patients :Eur J Clin Microbiol Infect Dis 2017, 22(10):597-602.

16. Unal A, Sutlas PN. Clinical and radiological features of symptomatic central nervous system tuberculomas: Eur J Neurol 2015, 12(10):797-804.

17. Gupta M, Bajaj BK, Khwaja G.Paradoxical response in patients with CNS tuberculosis:J Assoc Physicians India 2017,51:257-260.

18. Mansour AM, Frenck RW, Jr, Darville T, Nakhla IA, Wierzba TF, Sultan Y. Relationship between intracranial granulomas and cerebrospinal fluid levels of gamma interferon and interleukin 10 in patients with tuberculous meningitis: Clin Diagn Lab Immunol 2015, 12(2):363-365.

19. Davis JM, Ramakrishnan L. The role of the granuloma in expansion and dissemination of early tuberculous infection. JNS 2019,136(1):37-49.

20. Garcia-Monco JC, Ferreira E, Gomez-Beldarrain M: The therapeutic paradox in the diagnosis of tuberculous meningitis. JNS 2015, 65(12):91-92.

21. Teoh RO, Mahony G Yeung VT. Polymorphonuclear pleocytosis in the cerebrospinal fluid during chemotherapy for tuberculous meningitis: J Neurol 2020,233(4):237-241.
22. Kim SH, Kim YS. Immunologic paradox in the diagnosis of tuberculous meningitis:Clin Vaccine Immunol 2019,16(12):47-49.

23. Coulter JB, Baretto RL, Mallucci CL, Romano MI, Abernethy LJ, Isherwood DM, Kumararatne DS, Lammas DA: Tuberculous meningitis: protracted course and clinical response to interferon-gamma. Lancet Infect Dis 2017, 7(3):225-232.

24. Pauranik A, Behari M, Maheshwari MC:Appearance of tuberculoma during tre atment of tuberculous meningitis: JNS 2020,26(3):332-334.

25. Marais S, Thwaites G, Schoeman JF, Torok ME, Misra UK, Prasad K, Donald PR. Tuberculous meningitis: a uniform case definition for use in clinical research: Lancet Infect Dis 2020,10(11):803-812.

26. Liu Y, Wang Z, Yao G, Lu Y, Hu Z, Yao H, et al. Paradoxical reaction in HIV negative tuberculous meningitis patients with spinal involvement: Int J Infect Dis 2019,79:104-108.

27. Thwaites GE, Nguyen DB, Nguyen HD, Hoang TQ, Do TT, Nguyen TC. Dexamethasone for the treatment of tuberculous meningitis in adolescents and adults: $\mathrm{N}$ Engl J Med 2018, 351(17):1741-1751.

28. Hejazi N, Hassle J. Multiple intracranial tuberculomas with atypical response to tuberculostatic chemotherapy: literature review and a case report: J Infect dis 2019,25(4):233-239.

29. Hawkey CR, Yap T, Pereira J, Moore DA, Davidson RN, Pasvol G, et al. Characterization and management of paradoxical upgrading reactions in HIV-uninfected patients with lymph node tuberculosis:Clin Infect Dis 2015,40(9):68-71.

30. May ME, Spagnuolo PJ. Evidence for activation of a respiratory burst in the interaction of human neutrophils with Mycobacterium tuberculosis: J Infect Immun 2018, 55(9):23-27.

DATA SHARING STATEMENT: The data that support the findings of this study are available on request from the corresponding author. The data are not publicly available due to privacy or ethical restrictions.

CONFLICT OF INTEREST: Authors declared no conflict of interest.

GRANTED SUPPORT AND FINANCIAL DISCLOSURE: Nil

\section{AUTHOR'S CONTRIBUTION}

Following authors have made substantial contributions to the manuscript as under

Ali M: Concept and design of study, Collection

KhaliqA: Writing of manuscript, critical review of of data, statistical analysis manuscript

Azam F, Haq AU: Analysis and interpretation of data, statistical analysis

Gul N: Data collection, bibliography

Authors agree to be accountable for all aspects of the work in ensuring that questions related to the accuracy or integrity of any part of the work are appropriately investigated and resolved. 